\title{
ELLIPTIC FUNCTIONS AND NON EXISTENCE OF COMPLETE MINIMAL SURFACES OF CERTAIN TYPE
}

\author{
CHI CHENG CHEN
}

\begin{abstract}
It is proved that any complete minimal surfaces in $\mathbf{R}^{n}(n>3)$ with total curvature $-4 \pi$ is conformally equivalent to the complex plane or the punctured plane, just like the case in $\mathbf{R}^{3}$.
\end{abstract}

1. Introduction. It has long been observed that complex analysis has a deep and fundamental influence in the theory of minimal surfaces. The object of this paper is to give another concrete example of such application. In fact, we will use some basic properties of the elliptic functions to show that there exists no.complete minimal surface (immersed and orientable) of genus 1 in some euclidean space, with total curvature $-4 \pi$. (It has been shown by Chern and Osserman [3] that any complete minimal surface in euclidean space, with finite total curvature, is conformally equivalent to some compact Riemann surface punctured at a finite number of points.)

This work was initially motivated by trying to understand the complete minimal surfaces in $\mathbf{R}^{n}(n>3)$ with total curvature $-4 \pi$. Why $n>3$ ? Because Osserman [7] has shown that the only complete minimal surfaces in $\mathbf{R}^{3}$, with total curvature $-4 \pi$, are the catenoid and Enneper's surface. After analysing the Gauss-Bonnet type inequality given by Chern and Osserman [3], we were obliged to solve a problem similar to Hopf's conjecture for compact surfaces in $\mathbf{R}^{3}$ of genus 1 , with constant mean curvature. The problem is whether there exists a complete minimal surface of genus 1 in some euclidean space, with total curvature $-4 \pi$. By analysis of elliptic functions, we obtained a negative result. In fact, this result is equivalent to saying that any complete minimal surface in $\mathbf{R}^{n}$, with total curvature $-4 \pi$, is conformally equivalent to the complex plane or the punctured plane, just like the case in $\mathbf{R}^{3}$.

Finally, we would like to express our sincere gratitude to Professor Karl Otto Sthor, at IMPA, Rio de Janeiro, Brazil, for introducing us to the characteristic properties of the Weierstrass $\boldsymbol{P}$ function, and, to Professor Manfredo P. do Carmo, at IMPA, and to our colleague Professor Plinio Simões, for useful discussions on some technique details during the preparation of this paper.

2. Preliminaries.

(A) Minimal surfaces. Let $\vec{x}: M^{2} \rightarrow \mathbf{R}^{n}$ be a minimal immersion, where $M$ is an orientable differentiable 2-manifold. In terms of isothermal parameters $\left(\xi_{1}, \xi_{2}\right)$, the

Received by the editors November 2, 1978 and, in revised form, February 5, 1979.

AMS (MOS) subject classifications (1970). Primary 53A10; Secondary 30A68. $0002-9939 / 80 / 0000-0277 / \$ 02.25$ 
immersion is characterized by the following properties:

$$
\sum_{k=1}^{n} \phi_{k}^{2}(\zeta) \equiv 0
$$

where $\zeta=\xi_{1}+i \xi_{2}, \phi_{k}(\zeta)=\partial x_{k} / \partial \xi_{1}-i \partial x_{k} / \partial \xi_{2}, \vec{x}=\left(x_{1}, \ldots, x_{n}\right)$ and

(2.2) $\phi_{k}(\zeta)$ 's are analytic functions of $\zeta$,

(2.3) $\sum_{k=1}^{n}\left|\phi_{k}(\zeta)\right|^{2} \neq 0$ for all $\zeta$, and

(2.4) $\vec{x}(\zeta)=\operatorname{Re} \int_{\zeta_{0}}^{\zeta} \vec{\phi}(\zeta) d \zeta$

integrated along any path with $\zeta_{0}$ a fixed initial point, where $\vec{\phi}=\left(\phi_{1}, \ldots, \phi_{n}\right)$.

Geometrically (2.1) means the parameters $\left(\xi_{1}, \xi_{2}\right)$ are isothermal, $(2.2)$ means the immersion is harmonic in terms of isothermal parameters, and (2.3) means the regularity of the surface.

The Gauss map $g: M \rightarrow p^{n-1}(C)$ is defined by

$$
g(\zeta)=\left[\vec{\phi}_{1}(\zeta), \ldots, \vec{\phi}_{n}(\zeta)\right]
$$

in terms of homogeneous coordinates. In fact, $g(\zeta)$ represents the oriented tangent plane generated by $\partial \vec{x}(\zeta) / \partial \xi_{1}$ and $\partial \vec{x}(\zeta) / \partial \xi_{2}$. For details see [3].

(B) Elliptic functions. By a lattice in the complex plane $C$ we shall mean a subset of the form

$$
L=\left\{w=m w_{1}+n w_{2} \mid m, n \in Z\right\},
$$

where $w_{1}, w_{2}$ are two R-linearly independent vectors in $C$. An elliptic function, with respect to $L$, is a meromorphic function on $C$ which is $L$-periodic, i.e.

$$
f(z+w)=f(z)
$$

for all $w \in L$.

The well-known Weierstrass $P$ function, associated to $L$, is defined by

$$
P(z)=\frac{1}{z^{2}}+\sum_{w \in L^{\prime}}\left[\frac{1}{(z-w)^{2}}-\frac{1}{w^{2}}\right]
$$

with $L^{\prime}=L-\{0\}$, which is elliptic with poles of order 2 in $L$.

It is known that $[6$, p. 8]

(2.9) the field of elliptic functions, with respect to $L$, is generated by $P$ and $P^{\prime}$.

\section{Statement and the proof of the theorem.}

THEOREM. Let $S$ be a complete minimal surface in $\mathbf{R}^{n}$, given by $x: M^{2} \rightarrow \mathbf{R}^{n}$, with total curvature $C(S)=-4 \pi$. Then $S$ is conformally equivalent to the complex plane or the punctured plane.

Equivalently, there exists no complete minimal surface in $\mathbf{R}^{n}$ of genus 1 and with total curvature $-4 \pi$.

Proof. Since the surface is orientable, we use isothermal parameters to put a Riemann surface structure on the manifold $M$. Since $S$ has finite total curvature, it is known that [3] $M$ is conformally equivalent to a compact Riemann $W$ punctured at a finite number of points $p_{1}, \ldots, p_{r}, r \geqslant 1$; and the global differentials $\phi_{k}(\zeta) d \zeta$ are meromorphic at each $p_{j}$. 
Let $\gamma$ be the genus of $W, \chi$ the Euler characteristic of $M$, then $\chi=2-2 \gamma-r$. By a theorem of Chern and Osserman [3], we have

$$
C(S) \leqslant 2 \pi(x-r)=2 \pi(2-2 \gamma-2 r)
$$

with $C(S)=-4 \pi$; we can conclude that either

$$
\begin{aligned}
& \text { (i) } \gamma=0 \text { and } r=1 \text { or } 2 \text {, or } \\
& \text { (ii) } \gamma=1 \text { and } r=1 \text {. }
\end{aligned}
$$

Case (i) means that $S$ is conformally equivalent to $C$ or $C-\{0\}$. Therefore it rests to prove that the case (ii) can never happen.

Now assume $\gamma=1$ and $r=1$, then $M=W-\{p\}$ and the differentials, $\phi_{k}(\zeta) d \zeta$ are meromorphic at $p$. From $C(S)=-4 \pi$, we have that

(3.3) the total order of intersection of the image of the extended Gauss map $g$ : $W \rightarrow p^{n-1}(C)$, with each hyperplane in $P^{n-1}(C)$ which does not contain $g(W)$, is 2 [3].

Now let us look at the universal covering space $X$ of $W$, and pass the conformal structure of $W$ to $X$. Then $X$ is conformally equivalent to the complex plane. Therefore we have a conformal covering map

$$
\pi: C \rightarrow W
$$

and

$$
L=\pi^{-1}(p) \text { is a lattice in } C .
$$

Set

$$
\tilde{x}=x \circ \pi: C-L \rightarrow \mathbf{R}^{n} \quad \text { and } \quad \tilde{\phi}_{k}(z)=\frac{\partial \tilde{x}_{k}}{\partial \xi_{1}}-i \frac{\partial \tilde{x}_{k}}{\partial \xi_{2}}, \quad k=1, \ldots, n,
$$

where $z=\xi_{1}+i \xi_{2}$ is the global parameter of $C$. Then from (2.2), (3.3), (3.6), we see that

(3.7) $\tilde{\phi}_{k}(z)$ are elliptic functions, with respect to $L$, and with poles in $L$, and

(3.8) $\max _{k}$ \{orders of poles of $\tilde{\phi}_{k}$ at each $\left.w \in L\right\}=2$.

Therefore, from (2.9), each $\tilde{\phi}_{k}$ has to be of the form

$$
\tilde{\phi}_{k}=a_{k} p+b_{k}
$$

for some $a_{k}, b_{k}$ in $C$.

Now set $\tilde{\phi}=\left(\tilde{\phi}_{1}, \ldots, \tilde{\phi}_{n}\right), \vec{a}=\left(a_{1}, \ldots, a_{n}\right), \vec{b}=\left(b_{1}, \ldots, b_{n}\right)$.

Then from (3.9), we have

$$
\tilde{\phi}=P \vec{a}+\vec{b} \text {. }
$$

From (2.3), (2.9), (3.10) and the hypothesis $C(S)=-4 \pi$, we have that

$$
\vec{a} \text { and } \vec{b} \text { are linearly independent in } C^{n} \text {. }
$$

Now let $\vec{a}=\vec{\alpha}+i \vec{\beta}, \vec{b}=\vec{u}+i \vec{v}$ in real and imaginary parts. From (2.1), (2.9), (3.11) and the proof of our previous work [1], we have that

$$
\vec{\alpha}, \vec{\beta}, \vec{u}, \vec{v} \text { are linearly independent in } \mathbf{R}^{n} .
$$

Now from (2.4), we have that

$$
\tilde{x}=h_{1} \vec{\alpha}-h_{2} \vec{\beta}+\xi_{1} \vec{u}-\xi_{2} \vec{v},
$$


where $h_{1}$ and $h_{2}$ are the real and imaginary parts of the Weierstrass zeta function $\zeta(z)=\int_{z_{0}}^{z} P(z) d z$.

Since $\tilde{x}$ is doubly periodic in $C-L$, then from (3.12), we can conclude that the linear function

$$
\xi_{1} \vec{u}-\xi_{2} \vec{v}
$$

is doubly periodic in the $\left(\xi_{1}, \xi_{2}\right)$-plane which leads to a clear contradiction. Q.E.D.

4. Remarks. (1) By our result we can see that it would be interesting to classify the set of complete minimal surfaces in $R^{n}$ with total curvature $-4 \pi$. In fact, we know that the simply-connected ones lie in $\mathbf{R}^{6}$ and the doubly-connected ones lie in $\mathbf{R}^{5}$, which can be seen by a similar analysis of the Gauss map, as done in [1] and [2] (or see [4]).

(2) We can use the Weierstrass $P$ function to construct complete minimal surfaces of genus 1 and connectivity 1 (i.e. $r=1$ ) in $\mathbf{R}^{n}$ with finite total curvature. In fact $P^{\prime}(z) \vec{a}+P(z) \vec{b}, z \in C-L$, will induce a complete minimal surface of genus 1 and connectivity 1 in $C^{2}=\mathbf{R}^{4}$, with total curvature $-8 \pi$, where $\vec{a}$ and $\vec{b}$ are two suitably chosen vectors in $C^{2}$ (in order to avoid singularity).

(3) Thus, it would be very interesting to know whether we can use the Weierstrass $P$ function to construct complete minimal surfaces of genus 1 and connectivity $c(c \leqslant 3)$ in $\mathbf{R}^{3}$, so that they complement examples of different topological structure to the ones given by Klotz and Sario [5]. Several attempts have failed.

(4) From our result we can see that the Chern-Osserman inequality $C(S)<$ $2 \pi(\chi-r)$, at least in special cases, is not sharp in terms of the genus of the complete minimal surfaces. A natural task, therefore, would be to give a more precise description of the relationship between the total curvature and the topological structure of the surfaces. One can even associate this relationship to the dimensions of the ambient spaces.

Final note. It seems that we know the answers of the questions posed by Gackstatter [4] at the end of his paper.

(1) There do exist complete minimal surfaces of total curvature $-2 \pi$, and they are given in [1].

(2) There do exist complete minimal surfaces of total curvature $-4 \pi$, other than Enneper's surface and the catenoid. For example, the holomorphic curve associated to Enneper's surface and the example given in [2] for doubly-connected case.

(3) We can use complex polynomials to construct simply connected holomorphic curves lying fully in $C^{m}$. Then by the method of Calabi [0], the complete minimal surfaces isometric to those holomorphic curves lie between $\mathbf{R}^{m}$ and $\mathbf{R}^{2 m}$, and possess finite total curvature. Therefore their dimensions (in the sense of Gackstatter) are greater or equal to $m$. With $m$ arbitrary, we cannot expect an upper bound for all the dimensions of the class of complete minimal surfaces, with finite total curvature. 


\section{REFERENCES}

0. E. Calabi, Quelques applications l'analyse complexe aux surface d'aire minima, Topics in Complex Manifolds, Les Presses de L'Université de Montréal, Montréal, 1968.

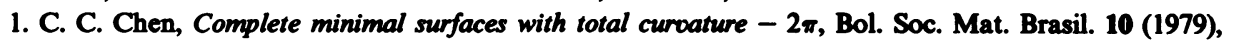
71-76.

2. __ A characterization of the catenoid, An. Acad. Brasil. Ci. 51 (1979), 1-3.

3. S. S. Chern and R. Osserman, Complete minimal surfaces in euclidean n-space, J. Analyse Math. 19 (1967), 15-34.

4. F. Gackstatter, Über die dimension einer minimalfläche und zur Ungleichung von St. Cohn-Vossen, Arch. Rational Mech. Anal. 61 (1976), 141-152.

5. T. Klotz and L. Sario, Existence of complete minimal surfaces of arbitrary connectivity and genus, Proc. Nat. Acad. Sci. U. S. A. 54 (1965), $42-44$.

6. S. Lang, Elliptic functions, Addison-Wesley, Reading, Mass., 1973.

7. R. Osserman, Global properties of minimal surfaces in $E^{3}$ and $E^{n}$, Ann. of Math. (2) 80 (1964), 340-364.

Instrtuto de Matemática e Estatística, Universidade de Sĩo Paulo, Sĩo Paulo, Brasil 